\title{
Una aproximación al pensamiento económico-normativo de John Rawls ${ }^{1}$
}

\author{
An Approach to John Rawls' Normative \\ Economic Though
}

\section{Uma aproximação ao pensamento econômico-normativo de John Rawls}

\author{
Diego Fernando Martínez Vallejo ${ }^{2}$ \\ Universidad Autónoma de Barcelona, Barcelona- España \\ diegofernando.martinez@e-campus.uab.cat
}

Recibido: 31.07 .14

Aprobado: 21.10 .14

1 Este artículo de reflexión se deriva del trabajo de grado en filosofía titulado Economía, sacrificio y envidia: las críticas de Jean-Pierre Dupuy a Una Teoría de la Justicia de John Rawls. Bogotá: Pontificia Universidad Javeriana, 2012. Agradezco a Roberto Solarte, el supervisor del trabajo de grado, y a Guillermo Hoyos (q.e.p.d.), cuya memoria persiste en muchos de nosotros. Agradezco finalmente a los dos evaluadores anónimos y sus útiles comentarios. Cualquier error, en todo caso, es mío y no involucra de ninguna manera a los aquí mencionados.

2 Magíster en Ciencia Política. Economista y filósofo. 


\title{
Resumen
}

Rawls, desde muy joven, estuvo interesado por los principios normativos del pensamiento económico moderno, y más específicamente su teoría del bienestar. Esta inquietud no sorprende. En primer lugar, a Rawls siempre le preocuparon la pobreza y la injusticia social, temas ampliamente discutidos en economía. Segundo, el pensamiento económico ha tenido un desarrollo muy cercano a la historia del utilitarismo, el gran oponente de Rawls. Este trabajo expone la apuesta económica de la teoría rawlsiana, la cual, a su vez, se muestra como una alternativa al pensamiento utilitario. Para ello, se hace hincapié en la insistente preocupación de Rawls por los menos aventajados, en la importancia que nuestro autor le da al mercado, y finalmente en su propuesta tributaria y salarial.

Palabras clave: John Rawls, Utilitarismo, Teoría de la Justicia, Principios de Justicia, Principio de la Diferencia.

Clasificación JEL: A12, B29, B31, B59, D63.

\begin{abstract}
Rawls, since an early age, was interested in the normative principles of the modern economic thought, particularly in its welfare theory. This concern is not surprising. First, Rawls was always concerned about poverty and social class injustice. Both issues widely discussed in economics. Second, the development of the economic thought has been very close to the utilitarian history, Rawls' greatest opponent. This paper presents the economic proposal of the Rawlsian theory, which in turn is shown as an alternative to the utilitarian approach. To do this, it emphasizes Rawls' persistent concern for the underprivileged, and the importance the author gives to the market, as well as his proposals on tax and wages.
\end{abstract}

Keywords: John Rawls, Utilitarianism, Theory of Justice, Principles of Justice, Difference Principle.

\section{Resumo}

Rawls, desde sua juventude, esteve interessado pelos princípios normativos do pensamento econômico moderno e em particular, pela teoria do bem-estar. Esta preocupação não surpreende. Em primeiro lugar, Rawls sempre esteve preocupado pela pobreza e pela injustiça social, questões amplamente discutidas na economia. Em segundo lugar, o pensamento econômico tem uma história de desenvolvimento muito próxima do utilitarismo, o grande adversário de Rawls. Este artigo apresenta a aposta económica da teoria de Rawls, a qual se mostra como uma alternativa ao pensamento utilitarista. Para esse fim, enfatiza a preocupação persistente de Rawls pelos desfavorecidos, a importância que o nosso autor dá ao mercado e, finalmente, sua proposta salarial e tributária.

Palavras-chave: John Rawls, Utilitarismo, Teoria da Justiça, Princípios da Justiça, Princípio da Diferença. 


\section{Introducción}

La obra de John Rawls, uno de los filósofos políticos más importantes del siglo $\mathrm{XX}$, tiene gran variedad de influencias, como incluso él mismo afirma cuando escribe que "de hecho no reclamo ninguna originalidad de las ideas que expongo" (Rawls 2002, 10). Su pensamiento, además de contener elementos claramente kantianos, recoge también un gran abanico de conceptos y categorías usados casi exclusivamente por la disciplina económica. Téngase presente que Rawls se interesó desde muy joven por la economía, asistiendo en varias ocasiones a seminarios de teoría económica, uno en 1949 con Jacob Viner y otro en 1950 orientado por William Baumol, y centrado en los textos Value and Capital y Foundations Economic Analysis de los afamados economistas John Hicks y Paul Samuelson, respectivamente. Así mismo, durante la primavera de 1951, con un grupo informal de estudio entre amigos, analizó los textos Elements of Pure Economics de Leon Walras y Theory of Games and Economic Behavior de John von Neumann y Oskar Morgenstern (Pogge 2010, 27).

Es frecuente la omisión y la falta de interés por esta parte de la vida de Rawls. Con todo, hoy son muchos los comentaristas que aluden a este período y a cómo su pensamiento se vio influenciado por la teoría económica; tal es el caso de Barry (1973), Dupuy (1998), Nagel (2003), Little (2014) y Pogge (2007; 2010). Recuérdese que entre sus principales lectores, las críticas de los economistas fueron siempre de las más agudas y oportunas; entre ellas no sobra mencionar las hechas por Sen (1982) y Arrow (1973) a las que Rawls responde en Liberalismo político. Con estos últimos autores, de hecho, Rawls había discutido un borrador de A Theory of Justice durante el año académico 1968-9, dos años antes de la publicación final del libro mientras dictaba con ellos un curso de filosofía política en Harvard (Sen 2010, 81). El interés de Rawls por la economía no sorprende. Primero, la disciplina económica ha concentrado gran parte de sus esfuerzos en los estudios de la pobreza y la justicia social. Segundo, la economía moderna, y más específicamente la economía de bienestar, es considerada por él como una extensión más del utilitarismo clásico, el gran adversario de Rawls.

El tema de la pobreza y de la injusticia preocupó siempre a Rawls, aunque su análisis lo hizo desde distintos campos y fue clara la distancia que asumió frente a la lectura económica. Rawls, por ejemplo, no estaba de acuerdo con que la abolición de la esclavitud fuera interpretada desde el análisis coste-beneficio, como lo hacía Fogel (1995). La libertad no podía estar supeditada a la eficiencia económica. Las intenciones de Abraham Lincoln, en opinión de Rawls, "fueron un compromiso entre la esperanza de justicia y los casi aplastantes obstáculos del mundo real" (Nagel 2003, 26). Rawls, por otra parte, atacó con dureza el utilitarismo, doctrina que ha influenciado enormemente el pensamiento económico moderno. El utilitarismo, nos recuerda Rawls, justifica el sacrificio de personas bajo el principio de la máxima utilidad (Rawls 2002, 35). De la misma manera, no toma en serio la distinción entre personas. Cuando la utilidad particular es agregada a un conjunto de utilidades, este criterio dice muy poco, o quizás nada, sobre las concepciones del bien que cada persona tiene, ni lo que cada una de ellas persigue (Rawls 2002, 38). 
Rawls advierte permanentemente sobre la cercanía del utilitarismo con la economía. Como bien lo expresa en diferentes obras $(2002,212)$, considera que es necesario recordar que el utilitarismo forma parte de una doctrina de la sociedad y que no es una forma de doctrina filosófica aislada. El utilitarismo ha incidido sobre aspectos fundamentales en varias disciplinas y ha adquirido un estatus casi hegemónico en una de ellas: la ciencia económica. Según él, "esto es explicable, en parte, porque si nos fijamos en los economistas más importantes de la tradición inglesa previos a 1900 y en los filósofos utilitaristas más conocidos, nos daremos cuenta que son las mismas personas" (Rawls 2009a, 212)3. Estas palabras, que escribirá en repetidas ocasiones en su libro Lecciones para la historia de la filosofía política (Rawls 2009a, 213, 480) así como en otros textos, incluido el prefacio a Una teoría de la justicia (Rawls 2002, 9; véase también Rawls 1981, v), demuestran su constante preocupación para que dicho vínculo no cayera en el olvido. De acuerdo con Rawls:

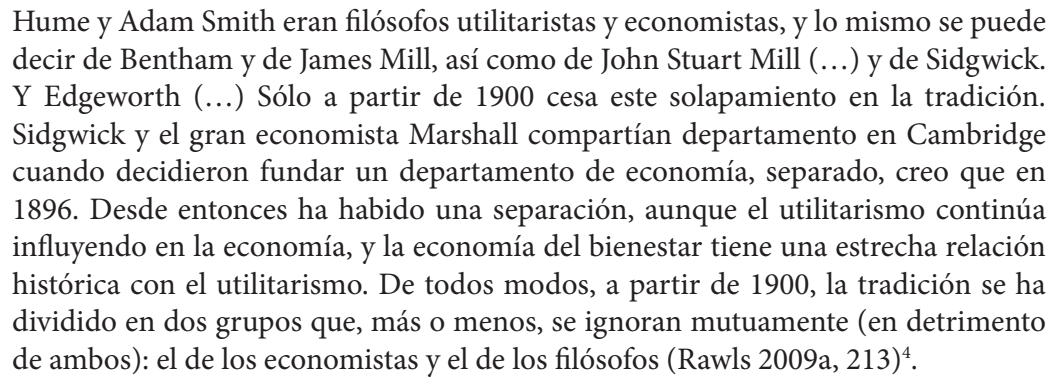

Rawls emprende una batalla en contra del utilitarismo y todas sus diferentes versiones. Entre ellas, sin lugar a dudas, la teoría utilitaria en economía. El objetivo de este artículo consiste en mostrar cómo Rawls enfrenta este adversario. Para ello, se analiza la crítica rawlsiana al criterio de elección utilitarista, ejemplificado en economía por el óptimo de Pareto. A su vez, muestra las distintas alternativas que se presentan frente a este último y con las cuales el filósofo de Harvard logra construir un pensamiento económico incipiente. En este sentido, este trabajo tiene la intención adicional de relacionar la obra de Rawls con la tradición de la economía política. A excepción de unas cuantas referencias, entre las que se destacan los trabajos de Little (2014) y de Dupuy (1998) —al que se sigue aquí de cerca-, y de los compendios de artículos editados por González y Pérez (2008), por un lado, y por Fleurbaey et al., (2010), por otro, no se

3 Exceptuando estas citas de Rawls que son textuales de sus Lecciones, las siguientes referencias de este libro se harán tomando la versión original en inglés.

4 Desde que Una teoría de la justicia fuera publicada, Rawls se vio sometido a duras críticas por incluir a Adam Smith dentro de la línea utilitarista (véase Dupuy 1998; Hurtado 2006; Sen 2010). Estas críticas son pertinentes. El espectador imparcial smithiano, al que Rawls se refiere en distintas ocasiones (Rawls 2002, 38), no es de ninguna manera utilitarista. La actitud de imponer políticas cuyo fin sea la maximización de la utilidad corresponde a la de un legislador soberbio, quien erige "su propio juicio como norma suprema del bien y el mal" (Smith 1997, 419, citado de Hurtado 2006, 12). Con todo lo anterior, Rawls parece haberse percatado de su error, y ya en sus Lecciones sobre la Historia de la Filosofía Moral utiliza el término “espectador juicioso" de Hume, y no el de "espectador imparcial" de Smith, ambos con significados distintos (Rawls 2000, 84). 
encontraron muchas referencias que trataran este tema. Si bien es cierto que Rawls fue muy modesto en pensarse como un autor de economía política y fue explícito en afirmar que su análisis concierne únicamente a los problemas de justicia (Rawls 2002, 249), es innegable que sus ideas sí reflejan intereses que lo vinculan con el pensamiento económico, al menos en lo concerniente a su aspecto normativo. Las alternativas que él presenta frente al utilitarismo entre las que se incluyen la postulación del principio de la diferencia, la concepción de un mercado regulado, y su propuesta salarial y tributaria, que se analizará más adelante, son, a todas luces, propuestas que cualquier economista identificaría dentro de su propio terreno.

\section{El utilitarismo clásico}

Para Rawls, el utilitarismo se clasifica en dos tipos: uno clásico, al que va esencialmente dirigida su crítica, y otro al que llama promedio. El primero de estos, si bien tiene como precursor a Jeremy Bentham, adquiere su formulación más clara y accesible en Sidwigck, precursor a su vez de las teorías de Francis Edgeworth, Arthur Cecil Pigou, Stanley Jevons, Vilfredo Pareto y León Walras (Rawls 2002, 34). Otros autores a los que Rawls presta atención dentro de este tipo de utilitarismo son John Harsanyi y Richard Brand, el primero centrado más en la economía y el segundo más en la filosofía (Rawls 2002, 34). La diferencia entre el utilitarismo clásico y el utilitarismo promedio, afirma Rawls, es que el clásico sostiene que "una sociedad está correctamente ordenada cuando sus instituciones maximizan el balance neto de satisfacción" (Rawls 2002, 35)5, contrario al utilitarismo promedio que dirige a la sociedad no a maximizar la utilidad total, sino la utilidad media de los individuos. En el caso de este último, cuyas figuras más importantes fueron David Hume y en cierta medida John Stuart Mill, se supone que cada hombre está en posición de ganar si se juzga a las ventajas a largo plazo cuando el derecho y el gobierno se adecúan a los preceptos fundados en la utilidad. Como bien dice Rawls, en Hume no se hace nunca referencia al hecho de que las ventajas de algunos sobrepasen las desventajas de otros (Rawls 2002, 43). En el caso del utilitarismo clásico, por el contrario, la maximización es pensada para el conjunto societario, no para el individuo. Rawls es un agudo crítico de esta forma de pensar en la toma de decisiones públicas, por lo que sus críticas van dirigidas en esencia a este tipo de utilitarismo. En palabras de Rawls, “al considerar estos contrastes (...), sólo he tenido presente la doctrina clásica, es decir, la opinión de Bentham y Sidgwick y la de los economistas utilitarios Francis Edgeworth, Arthur Cecil Pigou, Stanley Jevons, Vilfredo Pareto y León Walras (...)" (2002, 43).

Jeremy Bentham es el precursor más importante de esta corriente de pensamiento. Como bien anota Guisán (2008), es a causa de Bentham que se llenó de contenido "la vieja fórmula de la vida buena como vida feliz" (2008, 460). Para Bentham, cada persona busca el placer y evita el dolor: es la ley a la vez necesaria

5 En la edición del Fondo de Cultura Económica, citada en la bibliografía, se traduce "equilibrio neto" en lugar de "balance neto". Como sugerencia de un evaluador anónimo, preferí dejar balance en tanto se adecúa mejor a la obra original de Rawls. En A Theory of Justice, en efecto, se escribe "when its institutions maximize the net balance of satisfaction (...)" (Rawls 1999a, 24). 
y moral de toda acción. "Ellos solos [el placer y el dolor] han de señalar lo que debemos hacer, así como determinar lo que haremos" (Bentham 1789, 1; traducción propia). En este sentido, las mejores acciones son aquellas que procuran la mayor felicidad humana, incluso a costa del sacrificio de algunos, y a su vez aquellas que evitan el mayor dolor posible. El pensamiento de Sidgwick, expresado en sus famosos libros The Methods of Ethics, uno de los textos clásicos de la filosofía del siglo XIX, y en The Principle of Political Economy, el primer tratado de economía utilitarista (Rawls 2008, 393), es una clara continuidad de las ideas expresadas por Bentham, aunque evidentemente reformuladas de una manera distinta.

Sidgwick creía que el principio de utilidad se fundamentaba sobre la razón y no sobre un principio egoísta tal como supuso gran parte de la corriente utilitaria (Sidgwick 1981, 418). Afirmaba que la razón es capaz, por vía intuitiva, de reconocer los primeros principios morales, que a su vez se presentan como verdades evidentes (self-evident truths). Entre estas evidencias está el hecho de que la justicia solo tiene sentido si las personas se piensan como iguales (Dupuy 1998, 132). Junto con la igualdad abstracta de los hombres, o lo que Sidgwick llama principio de la equidad, se asume como evidente el principio de amor propio racional, el hecho de que todos los individuos tienen preferencias racionales que pueden ordenar de acuerdo con niveles de felicidad dados y, por tanto, comparables (Rawls 2008, 389-390). Supóngase ahora una totalidad matemática cuyos elementos son idénticos los unos a los otros. Supóngase además que estos elementos cuantifican, cada uno con el mismo valor, la felicidad del conjunto. Vista desde esta totalidad, la única regla que se justifica es la imparcialidad en el tratamiento de sus elementos. Así, el bien universal se define como la suma y resta de los placeres y dolores de los individuos, "e incluso de todos los seres capaces de tener sensaciones" (Rawls 2008, 396; Dupuy 1998, 133).

Bajo estos supuestos, Sidgwick considera que el principio de utilidad ha sido formulado con un grave error. La razón no busca la "felicidad del mayor número", como había supuesto parte del utilitarismo previo - particularmente Francis Hutcheson-, sino "la felicidad del conjunto". En consecuencia, los problemas de política pública habrán de decidirse en la medida que maximice la utilidad de la totalidad, independientemente del número de elementos que contenga o de su distribución (Rawls 2008, 408). Edgeworth, el buen alumno de Sidgwick en Cambridge, lleva al extremo tal afirmación, y propone una ciencia moral y matemática cuyo objeto de estudio sea la maximización de la utilidad (Edgeworth 1881, 1-4). Por ello, Rawls afirma que aunque Edgeworth era principalmente conocido como economista, también tenía una vertiente filosófica, al menos como filósofo moral (Rawls 2008, 162). El utilitarismo clásico, y más específicamente lo que Rawls llama la serie o trio BES - Bentham, Edgeworth y Sidgwick - (Rawls $2008,375,410$ ), sostiene así que la felicidad del conjunto social constituye la meta del vivir humano, y confiere sentido a los placeres y a los dolores, y a los sacrificios momentáneos que tienen valor moral en cuanto van encaminados a la consecución de un placer más intenso, vivo y profundo (Dupuy 1998, 133).

Para Rawls, esta teoría es peligrosa puesto que define el bien independientemente de lo que es justo. Esto, en palabras de Rawls, tiene dos significados: primero no toma en cuenta nuestros juicios de valor respecto a qué cosas consideramos como buenas (nuestras concepciones del bien), o las toma como una clase 
distinta de los juicios intuitivos, como aquello que dictaminan la maximización de la felicidad del conjunto. Segundo, para esta teoría la bondad de las cosas no hace referencia a lo que todos podemos pensar como justo (Rawls 2002, 36). La distribución correcta en cualquier caso es la que produce la máxima satisfacción (Rawls 2002, 38). Así, la distribución de las satisfacciones de la sociedad —entre ellas la riqueza - no tiene ningún sentido en el utilitarismo clásico. Si las distribuciones son inequitativas, incluso muy inequitativas, pero maximizan el nivel de satisfacción social, el utilitarismo clásico las justifica.

\section{Beneficios económicos diferenciados, pero bajo el cumplimiento de los derechos y las libertades básicas}

Rawls dejó claro desde un comienzo que su Teoría de la justicia es de naturaleza sumamente kantiana y que su objetivo principal era llevar la teoría del contrato social representada por Locke, Rousseau y Kant a un nivel mayor de abstracción (Rawls 2006, 10). Las similitudes de la teoría rawlsiana de la justicia con la filosofía kantiana son innegables. Su teoría, al igual que en Kant, supone una asociación de personas racionales que reconocen ciertas reglas de conducta como obligatorias. Así mismo, en ambos autores existe la necesidad de que los principios de justicia, aquellos que estamos dispuestos a reconocer como razonables, se establezcan sin hacer referencias a situaciones particulares - las llamadas inclinaciones en el lenguaje kantiano- tales como el estatus y la posición social, e inclusive las dotes naturales. Esto garantiza que los principios de justicia favorezcan en el mismo grado a todos, e impide que nadie tome ventaja debido a circunstancias contingentes.

Debe tenerse en cuenta que mientras el concepto de racionalidad aquí es interpretado "en el sentido estrictamente tradicional de la teoría económica, según la cual se emplean los medios más efectivos para fines dados" (Rawls 2002, 26) y en el que cada persona hace todo lo posible para promover sus propios intereses (Rawls 2002, 140), lo razonable se relaciona con aquellos principios que cualquier persona acordaría en una justicia imparcial, puntos comunes que suscitan el compromiso y la cooperación de todos (Rawls 2002, 30) ${ }^{6}$. Rawls establece así una distinción análoga a la que Kant (2002) previamente había formulado entre el imperativo categórico e imperativo hipotético, esto es, entre aquellas máximas que se asumen como leyes universales - lo razonable-, y aquellos imperativos que obligan a actuar de tal modo que pueda ser alcanzable algún fin propuesto - lo racional-. De acuerdo con esto, el sentido público de justicia no necesariamente es opuesto con la búsqueda del interés propio individual si este no contraviene los principios últimos de justicia (Rawls 2002, 18-19).

Rawls mantiene las mismas distinciones del contrato social kantiano, planteando así una justicia como equidad (fairness, también traducida como imparcialidad) en la cual todos están situados de manera semejante (Rawls 2002, 25).

6 Sen $(1988,10-22)$ subraya que si bien la racionalidad como maximización del interés propio es una concepción dominante en economía, no es la única. La racionalidad como consistencia interna, la cual evalúa la relación entre elecciones en situaciones distintas, también tiene gran peso en la historia de la disciplina (véase también Sen 2004, 19-20). 
Igualmente, y contrario al utilitarismo, donde el bien depende de los niveles de satisfacción e insatisfacción del conjunto societario, la justicia como equidad no concluye en la lógica del sacrificio en tanto todos los individuos asumen principios de justicia inviolables (Rawls 2002, 42). A pesar de la marcada influencia kantiana, Rawls añade varios aspectos (Rawls 2002, 237). Él cree, sin embargo, que estas adiciones "siguen bastante de cerca la doctrina de Kant, al menos cuando todos sus escritos éticos son considerados en conjunto" (Rawls 2002, 237). Entre estos aspectos, Rawls resalta el hecho de que los principios elegidos se aplican a "las instituciones sociales más importantes en un esquema de cooperación" (Rawls 2002, 62). En otras palabras, se aplican a cualquier sistema cuyas normas son el resultado de acuerdos erigidos públicamente y bajo la directa participación de todos (Rawls 2002, 63).

Subraya además que en el esquema de la justicia como equidad las personas son tanto racionales como mutuamente desinteresadas. Es decir, son personas que no solo buscan la consecución de sus propios fines de acuerdo con unos medios dados, sino que además se interesan fundamentalmente en sus propios asuntos (Rawls 2002, 238). Esto no quiere decir en absoluto que sean individuos egoístas, personas a las que solo les preocupa su propio bienestar, sino que se "les concibe como seres que no están interesados en los intereses ajenos" (Rawls 2002, 26). No importa, digámoslo así, si un católico quiere construir una parroquia si lo que quiere un musulmán es construir una mezquita. El concepto de persona moral se caracteriza por la facultad de actuar conforme a un sentido de la justicia que no impide que los hombres busquen racionalmente sus propias concepciones del bien, incluso si estas fueran consideradas como sus fines últimos (Gaiada 2008, 2). De aquí, precisamente, se garantiza lo que Rawls llama reciprocidad, una idea que se encuentra a medio camino entre la imparcialidad -la búsqueda de unos principios de justicia-y la idea de la ventaja mutua, en donde todos y cada uno de los societarios obtienen ciertos tipos de provecho y beneficio de los acuerdos establecidos (véase Rawls 2006, 68).

Dados estos supuestos, Rawls construye una situación hipotética en la cual todos los individuos son capaces de aceptar imparcialmente los mismos principios de justicia (Rawls 2002, 41). Esta posición original, como la llama Rawls, ha de estar caracterizada por un velo de ignorancia, en el que nadie sabe cuál es su lugar en la sociedad, su posición o estatus social; "nadie sabe cuál es su suerte en la distribución de ventajas y capacidades naturales, su inteligencia, su fortaleza" (Rawls 2002, 25). Las personas en la situación original, dice Rawls, aceptarían y reconocerían públicamente los principios elegidos, los cuales han de ser satisfechos por las instituciones sociales básicas (Rawls 2002, 26). Rawls expresa que los dos principios que se elegirían en un procedimiento así serían:

\footnotetext{
Primero: cada persona ha de tener un derecho igual al esquema más extenso de libertades básicas que sea compatible con un esquema semejante de libertades para los demás.

Segundo: las desigualdades sociales y económicas habrán de ser conformadas de modo tal que a la vez: a) se espere razonablemente que sean ventajosas para todos; b) se vinculen a empleos y cargos asequibles para todos (Rawls 2002, 67-68).
} 
Rawls supone que estos principios de justicia son los que implícitamente se afirmarían en la cultura pública de una sociedad democrática y, por tanto, representan un acuerdo establecido entre personas distintas, con doctrinas religiosas, filosóficas y morales diferentes (Rawls 1999b, 340). A esto Rawls lo denomina con el nombre de consenso entrecruzado, característica fundamental de las sociedades pluralistas. Estos principios no están, por ello mismo, sujetos a regateos ni a cálculo de intereses (Rawls 2002, 17). Dan poder, además, a que las personas tengan derecho a reclamar injusticias cuando observen que alguno de los principios ha sido violado. De ahí precisamente la concepción rawlsiana de ciudadanía. Siguiendo a Rawls, "el derecho a quejarse está limitado a las violaciones de principios (...). Una queja es una protesta dirigida de buena fe a otro. Proclama la violación de un principio que ambos individuos aceptan" (Rawls 2002, 206).

El primer principio otorga ciertas libertades básicas que cualquier persona tiene derecho como la libertad política (el derecho al voto y a ocupar cargos públicos), libertad de expresión y de asociación, las bases sociales para el respeto a uno mismo, la libertad de conciencia y de pensamiento, la protección de presiones psicológicas y de asalto físico, y el derecho a la posesión de propiedades personales. Entre estas últimas, sin embargo, no se incluyen los medios de producción, por lo que estos últimos han de ser tratados sin las garantías que otorga este principio.

El segundo principio se divide a su vez en dos. Mientras en la primera parte se postula el principio de la diferencia, en la segunda se enuncia el de la justa igualdad de oportunidades, el cual garantiza que todas las personas tengan las mismas oportunidades en el acceso a los empleos y cargos. Este principio, sin embargo, supone una redirección de los recursos públicos a la educación hacia la población más desfavorecida, incluyendo aquella que carece de capacidades fundamentales. Los más ricos, en este sentido, no solo deben cubrir los gastos que implica su enseñanza, sino que además se verán obligados a colaborar en la de aquellos menos afortunados (Rawls 2002, 104). El principio de la diferencia, por otra parte, hace referencia a que las desigualdades sociales y económicas solo podrán ser toleradas en caso de que beneficien a la persona menos aventajada.

La posición original establece igualmente una prioridad en los principios en caso de que estos den lugar a conflictos. El primer principio es prioritario al segundo, y dentro de este la igualdad de oportunidades es prioritaria al principio de la diferencia. Este procedimiento, que Rawls construye valiéndose de la teoría utilitaria en economía cuando esta describe preferencias de los agentes que muestran un orden lexicográfico, supone así que "el orden habrá de ser maximizado siempre y cuando los principios procedentes hayan sido plenamente satisfechos" (Rawls 2002, 53). La diferenciación económica solo puede ser tolerada, por tanto, si todos tienen las mismas libertades básicas y si existen de hecho oportunidades reales de acceso a empleos y cargos.

Para el principio de la diferencia, el orden lexicográfico tiene un doble sentido. En primer lugar, limita la posibilidad de que las ganancias privadas, a futuro, puedan coexistir con pérdidas de libertad o con desigualdad de oportunidades. Por pequeña que sea, ninguna ganancia económica puede compensar el cumplimiento de los derechos primarios, los cuales tienen un mayor valor en el esquema de prioridades sociales (Dupuy 1998). En segundo lugar, permite que las 
diferenciaciones económicas ya existentes puedan ser confrontadas por medio de una política fiscal progresiva en caso de que perjudiquen a alguno de los dos principios que le anteceden. De esto hablaremos más adelante y con mayor detalle.

\section{La eficiencia importa, pero sin perjuicio de los menos aventajados}

Los principios de la justicia, afirma Rawls, hacen que su teoría sea deontológica, ya que los individuos no actúan buscando maximizar el neto de satisfacción, sino solo por la estima de los principios. Como se dijo previamente, el principio de la diferencia, representado por la letra $a$, cuenta con la menor valoración en el orden de los principios rawlsianos. Pese a esto, es de sumo interés en Rawls al asumirse como la alternativa al óptimo paretiano - también llamado por él como principio de eficiencia-, y cuya postulación es clásica dentro del utilitarismo económico. El óptimo paretiano sostiene que una asignación es eficiente siempre y cuando cualquier cambio que beneficie a una persona necesariamente debe perjudicar a otra. En palabras de Rawls, el principio de eficiencia establece que "una configuración es eficiente siempre que sea imposible cambiarla de modo que beneficie a algunas personas, al menos una, sin que al mismo tiempo dañe a otras personas, al menos una" (Rawls 2002, 73, palabra y énfasis añadido)7.

El óptimo paretiano es un criterio de carácter normativo dentro de la ciencia económica, el cual plantea situaciones con cierta validez moral dentro de esta disciplina. Rawls ataca este principio con fuerza, denunciándolo como contraproducente tanto en términos de inequidad social y económica como en términos morales. Una sociedad en la que es válido que unos ganen mientras los más pobres pierden es una sociedad sumamente frágil. Además de afectar la estima de las personas - el respeto a uno mismo-, quizás el más importante de los bienes primarios, conlleva al surgimiento de envidias y de resentimientos; este último un sentimiento moral excusable en cuanto producto de una injusticia. Tanto la envidia como el resentimiento han sido ampliamente reconocidos como causantes de las más agudas violencias, volviendo así inestable y frágil el sistema (Rawls 2002, 480-484).

El principio de la diferencia, que Rawls formula como un ataque al criterio paretiano, establece por el contrario que las ventajas de los más favorecidos solo serán aceptadas si a su vez benefician a todos, con especial énfasis en el hombre menos aventajado. Como dice Rawls, "sólo es justificable si la diferencia de expectativas funciona en beneficio del hombre representativo peor colocado (...). La desigualdad en las expectativas es permisible sólo si al reducirla se empeora aún más a la clase obrera" (Rawls 2002, 83). No existe, por otra parte, ninguna contradicción entre el principio de la eficiencia y el principio de la diferencia. Nótese que cuando el principio de la diferencia es plenamente

7 La edición al español del Fondo de Cultura Económica tiene un grave error de traducción, ya que escribe "es eficiente siempre que sea posible" (énfasis añadido). El original de Rawls dice "The principle holds that a configuration is efficient whenever it is impossible to change it so as to make some persons (at least one) better off without at the same time making other persons (at least one) worse off" (Rawls 1999a, 58, énfasis añadido). 
satisfecho - cuando se han agotado todas las posibilidades de mejoramiento de la clase obrera- se alcanza un punto en el que cualquier otro beneficio de los más aventajados implica necesariamente el empeoramiento de la situación del más pobre (Rawls 2002). El principio de la diferencia, por tanto, es solo el único estado óptimo posible dentro de los muchos e infinitos puntos del espacio paretiano.

Aquí vale la pena mencionar que contrario a la interpretación que muchas veces se ha hecho de Rawls, sobre todo desde la economía misma (véase, por ejemplo, Mankiw 2009, 443, 444), el más desfavorecido acepta este principio no por algún tipo de temor o aversión al riesgo — que en caso de existir es totalmente desconocido gracias al velo de ignorancia supuesto por Rawls (2002, 135, 136) - Tal caso se asimilaría al modelo utilitarista de John Harsanyi (1953; 1955) basado en el supuesto de que los agentes minimizan su riesgo en momentos de incertidumbre. Este supuesto, sin embargo, no existe en Rawls. Como él mismo dice, "en la tesis de Harsanyi, existen otros aspectos muy diferentes y en los que utiliza la restricción para desarrollar una teoría utilitaria”, la cual Rawls busca rechazar (Rawls 2002, 135). El más desfavorecido, como los demás societarios, no toma estas decisiones aisladamente; son asociados que se comprometen los unos con los otros a respetar un contrato según principios definidos públicamente y aceptados unánimemente bajo el respeto mutuo. Rawls lo dejó claro en Liberalismo político, libro en el que explica que los repartos a los que hace referencia el principio de la diferencia no han de ser entendidos como meras medidas de bienestar psicológico o de utilidad (Rawls 2006, 183). Si fuera así, los más aventajados podrían objetar que los principios de justicia los perjudican si comparan sus beneficios respecto a sistemas previos e injustos. Pero la justicia como equidad, ni "ninguna concepción de justicia podría superar la idea de la ventaja mutua si se interpreta de esta manera" (Rawls 2006, 183).

\footnotetext{
Hagamos de cuenta que trasladamos a un número de personas de una sociedad, en la que la propiedad es muy desigual, producto de la suerte y la fortuna, a otra que, bien ordenada, se regula por los dos principios de justicia. No hay ninguna garantía de que todos salgan ganando con el cambio si juzgan la situación basados en actitudes previas (Rawls 2006, 41).
}

Ahora bien, en la teoría de la justicia rawlsiana las personas son representadas como personas morales, quienes pueden reconocer en ellas y en los otros contingencias arbitrarias. Como sujetos morales en el universo rawlsiano, las personas son capaces de cooperar en beneficio de todos, incluyendo el de aquellos menos favorecidos (Rawls 2002). "La justicia como imparcialidad es una teoría de nuestros sentimientos morales tal y como se manifiestan en nuestros juicios meditados" (Rawls 2002, 121), dice Rawls, y por esto es necesario que los principios que

8 El principio de la diferencia cambia sustancialmente de Una teoría de la justicia a Liberalismo político, en parte por las reacciones y críticas que se dieron a partir de la primera obra. Pasa de "Las desigualdades económicas y sociales (...) se espera razonablemente que sean ventajosas para todos" a "Las desigualdades económicas y sociales (...) deben ser para el mayor beneficio de los miembros menos aventajados de la sociedad (Rawls 2006, 36). Como vemos, en la segunda formulación, Rawls ya es explícito en afirmar que el principio debe necesariamente mejorar la posición "de los miembros menos aventajados de la sociedad". 
sean aceptados en la posición original desempeñen un papel no solo en nuestro razonamiento, sino también en nuestra conducta moral (Rawls 2002).

Rawls, de hecho, define su teoría de la justicia como una teoría de los sentimientos morales, la cual, como él mismo afirma, concuerda con el título de uno de los libros de filosofía moral más importantes del siglo XVIII: la Teoría de los sentimientos morales de Adam Smith, curiosamente considerado el padre de la economía moderna (Rawls 2002, 59). Esto no es casual. Recuérdese que gran parte de la ilustración escocesa, incluyendo a Hume y a Smith, reconocía que los hombres eran capaces de ponerse en el lugar del otro cuando, bajo un ejercicio de imaginación, experimentan cómo estarían ellos si estuvieran en la situación de los demás. Contrario a la teoría económica, alejada de ese sentimentalismo que la fundó y que pretende ser una ciencia hipotética-deductiva, la propuesta de Rawls es diferente. En palabras de Dupuy:

\footnotetext{
La cuestión es aquí la de un contrato social (...) [pero uno en el que] los societarios se hablan y se comprometen los unos con los otros. La promesa, el compromiso, el pacto son reguladores éticos y políticos (...) y juegan un papel de primer orden en la problemática de Teoría de la Justicia. La unanimidad rawlsiana no es pues la de aquellos a los que llegan, por el hecho de enfrentarse a un problema común, individuos racionales, separados y sin relaciones (Dupuy 1998, 151, palabras añadidas).
}

\section{El mercado importa, pero uno regulado y justo}

La teoría rawlsiana defiende con fuerza una economía basada en el mercado (Rawls 2002). Entre las bondades del mercado está el hecho de que $i$ ) los precios son determinados por la oferta y por la demanda (Rawls 2002, 254), ii) permite, contrario a los sistemas autoritarios, que la producción de bienes esté regulada por las preferencias de los consumidores, las cuales a su vez son reveladas por sus compras (Rawls 2002, 254), y iii) garantiza la justa igualdad de oportunidades y empleos. En el mercado, por ejemplo, las personas escogen libremente sus carreras y ocupaciones así como el lugar en el que desean ejercerlas, caso contrario a la dirección centralizada del trabajo, opuesta en cualquier caso a los principios de libertad (Rawls 2002, 255). Así mismo, iv), gracias a las virtudes de la tasa de ahorro y de inversión, "se expresa una gran preocupación por prevenir daños irreversibles y por administrar recursos naturales y conservar el ambiente" (Rawls 2002, 254). Finalmente, v) el mercado facilita la eficiencia y, por tanto, situaciones favorables para todos. Retomando la Teoría del Equilibrio General Walrasiano, el paradigma dominante en la teoría económica neoclásica, Rawls sostiene que la información suministrada por los precios lleva a que los agentes actúen de un modo tal que logran, dadas sus interacciones, escenarios ventajosos para todos respecto a situaciones previas (Rawls 2002, 255).

El mercado rawlsiano, sin embargo, es un mercado ideal, uno en el que el poder económico no está de tal manera concentrado que unos pocos individuos sean capaces de influir a su antojo en los precios. Por tanto, las restricciones monopólicas deben ser reconocidas, intervenidas y corregidas. De igual manera, en una economía de mercado justo, las empresas, sean privadas o estatales, dan por sentados los precios de los insumos, por lo que no entablan ni guerras de 
precios ni otras luchas por el poder (Rawls 2002, 255). En ambos casos, es claro que Rawls retiene de la teoría económica la noción de mercados perfectamente competitivos que, aunque se asocian a su tamaño y al número de oferentes, su principal característica es que los agentes aceptan, en cualquier transacción mercantil, los precios de los bienes y servicios como productos de la misma interacción social.

A pesar de las ventajas del mercado, Rawls deja claro que este ha demostrado ser también el causante de múltiples injusticias. "El que exista un sistema ideal de propiedad privada que sea justo no implica que las formas históricas sean justas o siquiera tolerables" (Rawls 2002, 257). Aunque es cierto que el mercado preserve el criterio de eficiencia, remunere el esfuerzo de los individuos por medio de salarios y ganancias, descentralice el poder económico, y aunque asegure en general un gran abanico de libertades, tiene la debilidad de ignorar las necesidades de las personas. Es por ello que para Rawls el Estado debe ser partícipe de los procesos económicos. Solo de esta manera es posible redistribuir las rentas a los menos aventajados. El esquema rawlsiano plantea así todo un sistema de impuestos y transferencias que permiten a los más pobres un mínimo de ingreso social razonable, asegurando con ello la plena satisfacción del segundo principio de justicia (Rawls 2002).

Los impuestos de herencia, de renta y de gasto en tasas proporcionales, e incluso en tasas progresivas cuando estas sean necesarias, son partes fundamentales para el cumplimiento de los principios de justicia. Mientras las tasas proporcionales de renta y gasto aseguran la justa igualdad de oportunidades en la enseñanza así como las transferencias que dictamina el principio de la diferencia, entre ellas diferentes formas de redistribución tales como subsidios al empleo y garantías de renta mínima (véase Van Parijs 2003), las tasas progresivas y los impuestos sobre la herencia cumplirán la función del mantenimiento de las instituciones y la conservación de la justicia con respecto al primer principio y al de la justa igualdad de oportunidad en los cargos. También se usarán las tasas progresivas, aunque solo en situaciones absolutamente necesarias, cuando se observen acumulaciones de riqueza intolerables capaces de corromper las instituciones (Rawls 2002, 261). Citando a Rawls:

\footnotetext{
Las diferencias en la distribución de la propiedad y riqueza que exceden lo que es compatible con la igualdad política han sido generalmente toleradas por el sistema legal (...). Así las desigualdades en el sistema socioeconómico, pueden minar cualquier igualdad política que hubiese existido en condiciones históricas más favorables. El sufragio universal da un contrapeso insuficiente, ya que cuando los partidos y las elecciones están financiados no por fondos públicos sino por contribuciones privadas, el foro político es tan influido por los deseos de los intereses dominantes que las medidas básicas necesarias para establecer una norma constitucional justa rara vez son debidamente presentadas (Rawls 2002, 214-215; véase también Pogge 2010, 32).
}

Una política fiscal suficientemente amplia para corregir las desigualdades podría sugerir un acercamiento de Rawls al pensamiento keynesiano. Las semejanzas entre Rawls y Keynes derivan de que para ambos las cargas impositivas son necesarias; para el primero en la medida en que garantizan la satisfacción de los principios de justicia, mientras que para Keynes son las bases para una política 
de pleno empleo. Sin embargo, Rawls afirma que su visión dista del pensamiento keynesiano, ya que este considera que "fue precisamente la desigualdad en la distribución de la riqueza la que hizo posible la rápida formación de capitales y el progreso más o menos continuo del nivel de vida en general". Para Rawls, "ciertamente hay medios más eficaces y justos de elevar el nivel de bienestar y cultura que el que Keynes describe" (Rawls 2002, 277). Rawls le critica al economista británico que su Teoría general del interés, el dinero y la ocupación pudo hacerse depender tan solo de mejorar la situación de la población menos favorecida, pero no lo hizo porque "en su sistema, no hay posibilidad real de suprimir, y mejorar, las condiciones de vida de los menos aventajados" (Rawls 2002, 278).

\section{Salarios y ganancias diferenciados, pero no por meritocracia}

Rawls advierte que no es posible establecer un vínculo esencial entre el mercado y los sistemas de propiedad privada sobre los medios de producción. Características propias del mercado como la asignación de precios por medio de tasas de interés e inversión, la fijación de precios a partir de equilibrios entre oferta y demanda, así como la libertad de cargos y ocupaciones se han dado tanto en sistemas socialistas como en economías capitalistas. El mercado, en este sentido, más que una característica propia del capitalismo, es una contingencia histórica de la que incluso los regímenes socialistas han sacado provecho (Rawls 2002, 254).

¿Cuál es entonces el sistema de producción que responde a las exigencias de justicia? Aunque Rawls afirma que si bien no puede con seguridad saberse cuál, "ya que depende en gran parte de las tradiciones e instituciones y fuerzas sociales de cada país, y de sus particulares circunstancias históricas" (Rawls 2002, 256), su esquema ideal de justicia utiliza los lineamientos del mercado bajo propiedad privada. La razón estriba en que los precios en el socialismo, argumenta Rawls, no cumplen una función distributiva ya que no corresponden al ingreso pagado a los individuos. La renta corresponde en su totalidad al Estado, siendo este el que decide cómo ha de repartirse. En los sistemas de mercado con propiedad privada, por el contrario, el precio se distribuye en salarios y ganancias privadas, no necesariamente iguales, cumpliendo así funciones distributivas. La distribución económica en la teoría rawlsiana está lejos de ser igualitaria. Para Rawls, cada quien ha de recibir lo que por derecho está en capacidad de exigir según los principios de justicia, y esto de ninguna manera implica igualdad económica.

Un sistema diferenciado de salarios tiene como ventaja principal garantizar la igualdad de oportunidades en un contexto en el que las cantidades de producción son reguladas por las decisiones de los consumidores. Ello permite crear todo un sistema de incentivos en aquellos sectores en los que "todos aceptan la conveniencia de un interés motivador, individual o colectivo, debidamente regulado por un sentido de la justicia" (Rawls 2002, 291). Salarios bajos en áreas concretas y de interés social o individual desincentivan la producción e innovación en perjuicio de todos y, por tanto, son indeseables. Por ello, la función del 
salario debe ser la de atraer "a las personas a los lugares donde más se les necesita desde un punto de vista social" (Rawls 2002, 291). Como dice Rawls:

\begin{abstract}
Supuestamente, dada la condición adicional en el segundo principio relativa a la asequibilidad de puestos y dado el principio de libertad en general, las mayores expectativas permitidas a los empresarios los estimulan a hacer cosas que aumentarán las expectativas de la clase laboral. Sus mejores perspectivas actúan como incentivos que harán más eficaz el proceso económico, más rápida la introducción de innovaciones, etc. (Rawls 2002, 83).
\end{abstract}

En la teoría de la justicia rawlsiana, sin embargo, los salarios y las ganancias no corresponden a lo que merecen los individuos gracias a sus dotes o talentos, como se asumiría en la meritocracia. Los salarios y las ganancias son producto de condiciones externas de oferta y demanda, y sirven como incentivos para asegurar la producción y el cubrimiento de aquellas necesidades sociales que los individuos reconocen como necesarias. Lo que las personas ganan, por tanto, no corresponde a un valor moral intrínseco y personal, sino a lo que por derecho las personas están autorizadas a exigir según los principios de justicia, lo que Rawls llama las "expectativas legítimas". Como "nadie merece una mayor capacidad natural ni tampoco un lugar inicial más favorable en la sociedad", tampoco "los más favorecidos por la naturaleza podrán obtener ganancia por el mero hecho de estar mejor dotados (...)" (Rawls 2002, 104, frase invertida).

Las capacidades naturales como aquellas contingentes son solo una muestra de las condiciones arbitrarias de los individuos, y por ello ningún valor moral puede vinculársele (Dupuy 1998, 195). Nacer dentro de una familia pobre o rica es solo cuestión de suerte — mas no así ha de entenderse el capitalismo-, lo mismo que con pocas o con muchas habilidades (Nagel 2003). Por ello, ni lo que se obtiene por la riqueza ni lo que se recibe por cuestiones meramente naturales puede suponer merecimiento alguno. Rawls, refiriéndose a San Agustín, aunque desde un punto de vista teológico pero que puede perfectamente extenderse a otros aspectos, será contundente en su A brief inquiry into the meaning of sin and faith al asumirse como un pensador con una postura radicalmente antimeritocrática (véase también Cohen y Nagel 2009):

(Una persona) cuanto más examina su vida, más se mira dentro de sí con completa honestidad y más claramente percibe que lo que tiene es una especie de regalo. Supóngase que esta persona es un hombre íntegro para los ojos de la sociedad, entonces se dirá a sí mismo: eres un hombre educado, sí, ¿pero quién pagó por tu educación? Eres un hombre bueno y correcto, sí, ¿pero quién te enseñó tus buenos modales y te proveyó de tu buena fortuna, gracias a la cual no has tenido que robar? Así que eres un hombre de una disposición amorosa y a quién no le gusta un corazón duro, sí, ¿ipero quién te enseñó el cuidado y el afecto que aprendiste cuando eras niño y con el que creciste para apreciar la bondad? ¿No deberías pues admitir que eres lo que has recibido? (Rawls 2009b, 240, traducción propia).

En la filosofía rawlsiana, los ciudadanos adquieren efectivamente determinados derechos cuando toman parte en acuerdos justos, "pero lo que están autorizados a exigir no es proporcional, ni depende de su valor intrínseco" (Rawls 2002, 287). Si bien es cierto que la distribución de los beneficios está en cierto 
modo relacionada con las capacidades de los individuos - lo que en teoría neoclásica se conoce como la productividad marginal del trabajo-, ni ellas ni el esfuerzo para desarrollarlas definen el ingreso que reciben las personas. En una economía competitiva los salarios son determinados por la oferta y la demanda -interacción que refleja las necesidades del mercado-, pero el valor moral de una persona no varía bajo estas contingencias, ni "de acuerdo con la cantidad de personas que ofrecen conocimientos similares, o de acuerdo con lo necesario de lo que él produzca" (Rawls 2002, 288). Nadie supone, por ejemplo, que los talentos de los cantantes se deterioren cuando no haya demanda de sus capacidades (Rawls 2002, 288). Sus ingresos, en efecto, pueden verse reducidos por cambios en el mercado - como en el caso de variaciones en las preferencias debido a epidemias o a catástrofes naturales-, pero dichos cambios no indican nada sobre las percepciones estéticas de la gente, ni mucho menos que estas hayan cambiado proporcionalmente a la variación del mercado.

La distribución de talentos, en este sentido, es vista como "un acervo común" con el que todos nos beneficiamos. Como comenta Dupuy, si bien es posible que las personas puedan educarse y adquirir competencias distintas según lo que el bien común demanda, con lo que se obtiene todo un sistema diferenciado de salarios y ganancias, nadie puede decir que estas sean merecidas, ni tampoco que sean el producto de sus cualidades intrínsecas. "Todo eso hace parte de la administración. La justicia está en otra parte" (Dupuy 1998, 196).

\section{Conclusiones}

Este trabajo pretende ser solo una aproximación al pensamiento económico de Rawls, el cual responde a la propuesta del autor de crear una alternativa a la doctrina utilitarista así como a todas sus múltiples versiones, incluida la teoría utilitaria en economía, también llamada economía del bienestar. Entre las apreciaciones de Rawls se destaca el principio de la diferencia, el cual establece la posibilidad de diferencias socioeconómicas siempre y cuando la ganancia de los más ricos también favorezca a los menos aventajados. Además de poner el acento en estos últimos, el principio de la diferencia asume una economía fiscal redistributiva basada principalmente en impuestos proporcionales al ingreso y al gasto. Los impuestos progresivos, por su parte, tienen una función menor, y su uso se limita únicamente en casos de absoluta necesidad. Por ejemplo, cuando las desigualdades económicas se han vuelto intolerables hasta el punto de corromper a las instituciones o poner en riesgo el cumplimiento de los principios de justicia. El principio de la diferencia también asume todo un sistema salarial y de ganancias diferenciado que sirve como incentivo para la producción y la innovación de aquellos sectores públicamente reconocidos como importantes.

La filosofía rawlsiana ha influenciado la economía normativa en gran medida. Su impacto en la misma, sin embargo, era de esperar. Primero, la economía moderna tiene una base clara en el utilitarismo, el gran adversario de Rawls. Segundo, la preocupación de Rawls por los más desfavorecidos hace que su teoría sea altamente aplicable, sobre todo hoy cuando las desigualdades económicas han adquirido matices casi desbordantes. El pensamiento de Amartya Sen o de Phillipe Van Parijs, autores con enorme aceptación en los círculos intelectuales 
de la disciplina económica, son solo unos de los muchos ejemplos. Sen, quien nunca se ha cansado de admitir la gran admiración que siente por el filósofo de Harvard, afirmaba que cuando leía a Rawls captaba el sentimiento que Wordworth describía en sus poemas: "era una dicha estar vivo en ese amanecer/ pero ser joven era estar en el cielo" (Sen 2010, 82).

Este artículo aboga porque el pensamiento de Rawls no sea solo cuestión de filósofos - para quienes sin duda alguna es uno de los grandes pensadores del siglo XX- Rawls tiene muchas cosas qué decir también a los economistas, formados actualmente bajo estructuras curriculares fundamentadas en la economía utilitaria, doctrina ampliamente aceptada en la mayor parte de las universidades de todo el mundo. Rawls tiene ya un lugar ganado, aunque muy pequeño, en el pensamiento económico; lugar que esperamos, no caiga hoy en el olvido. Su reflexión muy seguramente puede abrir luces a nuevas formas de pensar la economía en tiempos de crisis y de desigualdad persistente.

\section{Referencias bibliográficas}

Arrow, Kenneth. «Some Ordinalist-Utilitarianism Notes on Rawls' Theory of Justice». Journal of Philosophy, Vol. 70, 1973: 245-263.

Barry, Brian. The Liberal Theory of Justice: A Critical Examination of the Principal Doctrines in A Theory of Justice by John Rawls. Oxford: Oxford University Press, 1973.

Bentham, Jeremy. The Principles of Moral and Legislation. Londres: Oxford University Press, 1789.

Cohen, Joshua, y Thomas Nagel. «Introduction to "A Brief Inquiry into the Meaning of Sin and Faith"». En A Brief Inquiry into the Meaning of Sin and Faith, de John Rawls, 1-23. Cambridge: Harvard University Press, 2009.

Dupuy, Jean-Pierre. El sacrificio y la envidia. El liberalismo frente a la justicia social. Traducido por Juan Gutiérrez. Barcelona: Editorial Gedisa, 1998.

Edgeworth, Francis. Mathematical Psychics. An Essay on the Application of Mathematics to the Moral Sciences. Londres: Kegan Paul, 1881.

Fleurbaey, Marc, Maurice Salles y John Weymar. Justice, Political Liberalism, and Utilitarianism. Themes from Harsanyi and Rawls. Cambridge: Cambridge University Press, 2010.

Fogel, Robert. Time on the Cross: The Economics of American Negro Slavery. Nueva York: W. W. Norton and Company, 1995.

Gaiada, María. «La posición original de Rawls bajo la luz kantiana». En VII Jornadas de Investigación en Filosofía para docentes, graduados y alumnos, 1-15. La Plata: Universidad Nacional de la Plata, 2008.

González, Jorge, y Mauricio Pérez. Pluralismo, legitimidad y economía política. Ensayos críticos sobre la obra de John Rawls. Bogotá: Universidad Externado de Colombia, 2008.

Guisán, Esperanza. «El utilitarismo». En Historia de la ética, 2. La ética moderna, Volumen 2, editado por Victoria Camps, 458-497. Madrid: Crítica, 2008.

Harsanyi, John. "Cardinal Utility in Welfare Economics and in the Theory of Risk-Taking». Journal of Political Economy, Vol. LXI, 1953: 434-435. 
"Cardinal Welfare, Individualistic. Ethics and Interpersonal Comparisons of Utility». Journal of Political Economy, Vol. 63, n 4, 1955: 309-321.

Hurtado, Jimena. «El sistema de Adam Smith: una alternativa liberal olvidada por John Rawls». Documentos CEDE, n 17, 2006: 1-22.

Kant, Immanuel. Fundamentación para una metafísica de las costumbres. Traducido por Roberto Aramayo. Madrid: Alianza Editorial, 2002.

Little, Daniel. «Rawls and Economics». En A Companion to Rawls, editado por Jon Mandle y David Reidy, 504-525. Chichester: Wiley-Blackwell, 2014.

Mankiw, Gregory. Principios de economía (Cuarta edición). Madrid: Parainfo S.A., 2009.

Nagel, Thomas. «La compasión rigurosa de John Rawls: una breve biografía intelectual». Revista Praxis, $n^{\circ}$ 16, 2003: 25-40.

Pogge, Thomas. John Rawls: His Life and Theory of Justice. Nueva York: Oxford University Press, 2007.

. "John Rawls: una biografía». Revista Co-herencia, Vol. 7, n 12, 2010: $13-42$.

Rawls, John. «The Method of Ethics (foreword)». En The Method of Ethics, de Henry Sidgwick, i-vi. Indianapolis: Hackett Publishing, 1981.

. A Theory of Justice. Cambridge: Harvard University Press, 1999a.

Collected Papers. Cambridge: Harvard University Press, 1999b.

Lectures on the History of Moral Philosophy. Cambridge: Harvard University Press, 2000.

- Teoría de la justicia. Traducido por María Dolores González. México

D.F.: Fondo de Cultura Económica, 2002. tica, 2006.

. Liberalismo político. Traducido por Segio Rene Madero. Barcelona: Crí-

. Lectures on the History of Political Philosophy. Cambridge: Harvard University Press, 2008.

. Lecciones sobre historia de la filosofía política. Traducido por Andrés de Francisco. Barcelona: Paidós, 2009a.

. A Brief Inquiry into the Meaning of Sin and Faith. Cambridge: Harvard University Press, 2009b.

Sen, Amartya. Choice, Welfare and Measurement. Oxford: Oxford University Press, 1982.

. On Ethics and Economics. Londres: Blackwell Publishing, 1988.

. Rationality and Freedom. Cambridge: Harvard University Press, 2004. La idea de la justicia. Traducido por Hernando Valencia. Madrid: Taurus, 2010.

Sidgwick, Henry. The Methods of Ethics. Indianapolis: Hackett Publishing Company, 1981.

Smith, Adam. La teoría de los sentimientos morales. Traducido por Carlos Rodríguez. Madrid: Alianza Editorial, 1997.

Van Parijs, Philippe. «Difference Principle». En The Cambridge Companion to Rawls, editado por Samuel Freeman, 200-240. Cambridge: Cambridge University Press, 2003. 